\title{
Alcohol detoxification in Ysbyty Gwynedd: Two small sips or one big gulp? Two-step screening more reliable for identification of alcohol dependency syndrome at risk of delirium tremens for routine care
}

\author{
Muhammad Salman, Christian Subbe \\ United Kingdom, Wales
}

\begin{abstract}
Compliance with pathways for hospitalised patients with alcohol dependency syndrome is often poor. A pathway for recognition and treatment of alcohol dependency was redesigned as part of a 12 month service improvement project in the acute medical unit using plan, do, study, act (PDSA) cycles.
\end{abstract}

A needs assessment was undertaken: Audit data from 2013 showed over-prescription of chlordiazepoxide for detoxification treatment (DT) leading to prolonged hospital admissions with an average length of stay of 5.5 days in 2012/2013.

Acceptability of screening tools was tested: Common screening tools (CEWA, AUDIT) were rejected by junior doctors due to the high number of questions as too cumbersome for routine practice. Compliance with usage in random samples over a three month period was persistently $<10 \%$. Testing of an abbreviated AUDIT questionnaire with only two questions and a specified threshold showed a AUROC of 1 ( $p<0.001$ for correct identification).

The screening tool was implemented in several PDSAs cycles. After the final cycle a random sample of 100 patients was reviewed for pathway compliance over a three months period. Eighty-six patients were screened with the two-question tool of these 18 were identified as possible risk. Of these 16 patients had the full AUDIT questionnaire, only eight with elevated values were started on DT. Overall compliance with the pathway increased to $84 \%$.

\section{Problem}

Alcohol-related hospital admissions are increasing.[1] There are a number of reasons for this, including the easy access, affordability, and the acceptance of a culture where drinking alcohol from an early age through to old age is becoming an acceptable behaviour.[4,5] According to the US Substance Abuse and Mental Health Services Administration's (SAMHSA's) National Survey on Drug Use and Health, 23.5 million persons aged 12 or older needed treatment for an illicit drug or alcohol abuse problem in 2009 alone. These represent 9.3 percent of persons aged 12 or older. Of these, only 2.6 million ( 11.2 percent of those who needed treatment) received it at a specialty facility.[17]

Different alcoholic beverages contain varying quantities of alcohol. A daily intake of more than $60 \mathrm{~g}$ of alcohol in men and $20 \mathrm{~g}$ in women significantly increases the risk of cirrhosis.[4]

More than four drinks per day is considered heavy alcohol use for women, and for men this is five drinks per day.[4] There is no absolute number of drinks per day or quantity of alcohol that defines alcoholism.[5] Alcohol dependence and harmful alcohol use are associated with increased risk of physical and mental health comorbidities including gastrointestinal disorders (in particular liver disease), neurological and cardiovascular disease, depression and anxiety disorders and ultimately, premature death.[5-7]
Brief interventions can be effective in reducing drinking in hazardous and harmful drinkers, but people with alcohol dependence and some harmful drinkers will require more specialist alcohol services. Alcohol misuse is also an increasing problem in children and young people, with over 24,000 treated in the NHS for alcohol-related problems in 2008 and 2009. Current practice across the country is varied, and access to a range of specialist alcohol services varies as a consequence. This quality standard describes markers of high-quality, cost-effective care that, when delivered collectively, should contribute to improving the effectiveness, safety and experience of care for harmful drinkers and people with alcohol dependence.[5]

Different tools are available for assessing alcohol abuse and detoxification.[1,2] Tools such as the Clinical Institute Withdrawal Assessment for Alcohol (CIWA) and the Alcohol Use Disorders Identification Test (AUDIT) questionnaires are recommended in the NHS across England and Wales,[2,4] and are expected to reduce alcohol-related hospital admissions and readmissions to hospital.[6]

The aim of this quality improvement project was to introduce an effective and efficient tool for scoring alcohol dependency in order to reduce unnecessary detoxifications and initiate appropriate referrals to support services.

\section{Background}


A local audit from 2012 showed bed usage in our local unit for admissions with conditions related to alcohol excess of 6778 days. These included head injuries, gastro-intestinal bleeds, and pancreatitis. A separate review of ICD-10 codes for patients admitted to the Ysbyty Gwynedd from September 2012 to September 2013 with ICD codes denoting alcohol detoxification registered 55 admissions. The mean length of stay of these patients was 5.5 days, with an age range of 21 to 64 years.

The National Confidential Enquiry for Patient Outcome and Death (NCEPOD) [14] issued a report in 2013 ('Measuring the Units') that columinates in the statement, "There was a failure to screen adequately for harmful use of alcohol and even when this was identified, patients were not referred for support."

The National Institute for Health and Care Excellence (NICE) has issued guidance on the diagnosis, assessment and management of harmful drinking and alcohol use disorders (NICE 115).[4] In particular, NICE recommends to "use formal assessment tools to assess the nature and severity of alcohol misuse, including the AUDIT for identification and as a routine outcome measure (see attached), SADQ or LDQ for severity of dependence, Clinical Institute Withdrawal Assessment of Alcohol Scale, revised (CIWAAr) for severity of withdrawal, and APQ for the nature and extent of the problems arising from alcohol misuse."

Appropriate treatment of alcohol withdrawal (AW) can relieve the patient's discomfort, prevent the development of more serious symptoms, and forestall cumulative effects that might worsen future withdrawals. Hospital admission provides the safest setting for the treatment of AW, although many patients with mild to moderate symptoms can be treated successfully on an outpatient basis. Severe AW requires pharmacological intervention. Although a wide variety of medications have been used for this purpose, clinicians disagree on the optimum medications and prescribing schedules. The treatment of specific withdrawal complications (such as delirium tremens and seizures) present special problems and requires further research.[8,9]

See supplementary file: ds4777.pdf - "Audit Questionnaire "

\section{Baseline measurement}

A review of the care pathway of patients admitted to the department of internal medicine at the Ysbyty Gwynedd in Bangor was carried out. The Ysbyty Gwynedd is a district general hospital with an average of 33 admissions per 24 hour period and 500 beds. The majority of patients are admitted through the 30 -bed acute medical unit.

An audit of clinical records showed that alcohol related data were only rarely entered. The admissions proforma contained data items to record the number of units of alcohol drank per week. These were rarely filled and do not comply with recommendation NICE CG115. There were no screening tools used for assessing the risk associated with alcohol excess and thus determining and identifying those patients at high-risk of delirium tremens.
Ancedotal evidence suggested that patients were started on alcohol detox inappropriately because of a lack of quantification of the alcohol excess and the associated risk. No patients were referred to the alcohol-liaison services in the community in a three months observation period. A patient's own involvement and the perception of being "ready for change" might hold the key to reduce the hospital admission rates.

\section{Design}

Time frame: Over a 12 month period from September 2013 to December 2014 the authors reviewed medical notes in the acute medical unit (AMU) and implemented the intervention.

Setting: The AMU has 24 beds with a six-bedded trolley bay and admits on average 33 patients over a 24 hour period from general practitioners and the emergency department. Patients are seen by a junior doctor at senior house officer and foundation year one level, and are subsequently reviewed by a consultant acute physician (during office hours) or a consultant general physician.

Process: At the outset of the project the authors reviewed 20 clerking proformas on the acute medical unit and a gastroenterology ward. While the majority of notes had comments about drinking, no information was present on the review of any of the 20 sets of notes about quantifying the risk of delirium tremens or the need for alcohol detoxification using any of the tools mentioned above. Samples of clerking proformas were altered for each of the Plan, Do, Study, Act (PDSA) cycles on Wednesday afternoons. On the subsequent Thursdays the proformas from the previous evening and night shift were reviewed and compliance against pre-agreed standards were measured.

Weekly ward-round were organised, and these were aimed at medical admissions with alcohol problems. The medical documentation was reviewed. Patient's alcohol dependency was assessed and drinking habits were reviewed with patients along with their keenness to seek help, support and ultimately to quit. Only two in every three admissions had an initial assessment for alcohol dependence.

\section{Strategy}

Three PDSA cycles were undertaken:

- PDSA cycle 1: Addition of the AUDIT questionnaire to the clerking
pro-forma

- PDSA cycle 2: Development of a shortened version of the AUDIT questionnaire

- PDSA cycle 3: Implementation of referral pathway to drug and alcohol services.

See supplementary file: ds4779.jpg - "Audit questionnaire values and prescribed detoxification regime" 


\section{Post-measurement}

PDSA cycle 1: The AUDIT questionnaire was added to the admission proformas. Admitting core medical trainees and foundation year one doctors were instructed to complete the questionnaire and start an alcohol detox regime only if indicated. Version one (usage of the full AUIDT questionnaires) were rejected by junior doctors due to the high number of items as too cumbersome for routine practice. Compliance with usage in random samples over a three months period was persistently less than 10 to $15 \%$.

PDSA cycle 2: Development of a shortened version of the AUDIT questionnaire. The AUDIT questionnaire is a 10 item tool. A casecontrol study was carried out to test out sensitivity and specificity of individual items for ability to discriminate between patients at high risk of alcohol dependency and withdrawal and patients with low risk. Out of the cohort of patients who had AUDIT questionnaire we selected 20 patients in the acute medical unit and to 20 patients with alcohol related liver disease in the gastroenterology unit. Verbal consent was taken from all patients.

Questions 3 and 4 of the AUDIT questionnaire had the highest sensitivity and specificity to alcohol dependence $(p<0.001)$. The testing of an abbreviated AUDIT questionnaire was based only on the two items and a specified threshold showed an area under the receiver operator characteristic curve of $1(p<0.001$ for correct identification of cases). While balancing the likely reduced sensitivity and specificity of the shorter tool against the risk of poor compliance of junior doctors with the longer screening tool, an abbreviated version was added to the admissions proforma. Hospital admissions were then screened with the two questions assessed in the medical clerking proformas. A total score of 3 points in question 3 and 4 was chosen as the cut-off for screening with the full AUDIT questionnaire. Teams were educated to use high scores in the full AUDIT questionnaire and start an alcohol detoxification regime to prevent delirium tremens.

A further random sample of 100 patients was sampled for pathway compliance over three months. There was evidence that 86 patients had been screened with the two-question tool. Of the latter 18 were identified as patients at possible risk. Of these 16 patients had the full AUDIT questionnaire, only eight had elevated values and were subsequently started on treatment to prevent delirium tremens. Overall compliance with the pathway increased to $84 \%$ (figure 2 audit values and need for detoxification).

PDSA cycle 3: Implementation of referral pathway to drug and alcohol services. The referral pathway was agreed with stakeholders (see above). Patients who screen positive on the twoitem questionnaire should have the complete AUIDT questionnaire administered. Patients with a value of 11 to 15 get referred to CAIS services, patients with a score of more than 15 get referred to Alcohol and Drug Liason services. In order to improve reliability one of the authors (MS) collected once a week data on the number of patients started on detoxification and the rate of referrals. appropriateness of alcohol detoxification and referral to support services accordingly: Patient with clinical needs who had not been referred to support services were referred on this occasion. Data from these rounds underpinned the business case for a alcoholliasion nurse. Due to sickness of the post-holder further progress has been delayed.

See supplementary file: ds4780.jpg - "Compliance after second PDSA cycle increases to $84 \% "$

\section{Lessons and limitations}

What the project has shown: Acceptability of a shorter screening tool was higher and lead to better compliance. In order to make pathways acceptable to a heterogeneous group of doctors and nurses requirements need to be simple and obvious. The authors believe that the resulting pathways represents an improvement over and above published evidence.

What others have shown: NCEPOD 'Measuring the Units' showed a low uptake of screening for alcohol dependency in a large sample of UK hospitals. There is therefore an opportunity for improvement. The shortened screening tool that we used has only got two items. Alternatively the AUDIT-C questionnaire [14] is also a two-item screening tool that could be used in a comparable way. The twoquestion based AUDIT questionnaire encourages compliance and is easy to use in the settings of an acute medical unit. Applied in practice it has helped to limit the number of patients with inappropriate prescription of alcohol detoxification regimes.

Strengths and weaknesses: The present project is only work from a single centre and make-up of services might differ in other hospitals. In particular, dedicated in-hospital case workers for patients with alcohol dependency might improve compliance and referral rates in a more cohesive manner than our pathway. The strength of our work is its simplicity and co-design with users (ie junior doctors). This is a critical step for any sustainable improvement.

Clinical Implications: NCEPOD 'Measuring the Units' showed poor compliance with usage of alcohol withdrawal scale in less than $10 \%$ of patients at risk. One underlying reason might be the time consuming nature of the usual screening tools. A shorter tool might have worse statistical properties but paradoxically still result in better clinical care for the majority of patients at risk.

Future research: It would be useful to know long term outcomes of patients screened to determine which patients are likely to successfully undergo treatment of alcohol dependency in a rehabilitation program. The systematic application of a 'Readiness to change' [15] tool might support this. Additionally the review of readmission and predictors for the same [16] would add a financial argument for targeting subgroups of patients with alcohol dependency syndrome.

\section{Conclusion}


The authors started in the absence of screening tool applied in assessing patients who were admitted to hospital. As a result, some patients received detoxification with chlordiazepoxide inappropriately, with a potential increase to the number of hospital admissions and bed days. The AUDIT and CIWA scores were rejected by our juniors. At the same time they assisted with the development of a two-question screening tool.

The authors also believe that closer involvement of community services like the alcohol and drug misuse team and CIAS in a patient's care will add in further reducing the readmission rate and relapses. What is needed now is the follow-up of patients referred to the alcohol and drug misuse services in the community and the CIAS in the community and monitoring of readmission rates.

The patient's own involvement and the perception of being "ready for change" might hold the key to reduce the hospital admission rates, although this will require further exploration!

\section{References}

1. Saunders JB, Aasland OG, Babor TF, de la Fuente JR, Grant M. Development of the Alcohol Use Disorders Identification Test (AUDIT): WHO collaborative project on early detection of persons with harmful alcohol consumption. II. Addiction 1993; 88: 791-804.

2. AUDIT;The Alcohol Use Disorder Identification Test.Guidlines for use in Primary Care. Second edition. WHO.Department of Health and Substance Dependance. J Psychopharmacol 2004;18(3):293-335

3. Hartsell Z, Drost J, Wilkens JA, Budavari AI. Managing alcohol withdrawal in hospitalized patients. JAAPA 2007 Sep;20(9):20-5

4. NICE (2011) Alcohol dependence and harmful alcohol use - audit support. NICE clinical guideline 115.

5. NICE (2010) Alcohol-use disorders: physical complications - audit support. NICE clinical guideline 100.

6. NICE (2010) Alcohol-use disorders: preventing harmful drinking - audit support. NICE public health guidance 24 .

7. Abott JA, Quinn D, Knox L. Ambu- latory medical detoxification for alcohol. Am J Drug Alcohol Abuse 1995;21(4):549-63.

8. American Psychiatric Association Task Force. Treatment of Psychiatric Disorders. Washington, DC: American Psychiatric Association, 1989. 187.

9. Anton RF, Becker HC. Pharmacology and pathophysiology of alcohol withdrawal. In: Kranzler, H.R. Handbook of Experimental Pharmacology: Volume 114. The Pharmacology of Alcohol Abuse. New York: SpringerVerlag, 1995. 315-67.

10. Brown ME, Anton RF, Malcolm R, Ballenger JC. Alcohol detoxification and withdrawal seizures: Clinical support for a kindling hypothesis. Biological Psychiatry 1988; 23:507-14.

11. Hasyashida M, Alderman Al, Mclellan T. Comparative effectiveness and costs of inpatient and outpatient detoxification of patients with mild-to-moderate alcohol withdrawal syndrome. New Engl J Med 1989; 320(6):358-65.
12. Institute of Medicine. Prevention and Treatment of Alcohol Problems. Washington, DC: National Academy Press, 1990. 268-9.

13. Kaim SC, Klett CJ, Pothfeld B. Treatment of the acute alcohol withdrawal state: A comparison of four drugs. Am J Psychiatr 1969; 125:1640-6.

14. Bush K, Kivlahan DR, McDonell MB, et al; The AUDIT alcohol consumption questions (AUDIT-C): an effective brief screening test for problem drinking. Ambulatory Care Quality Improvement Project (ACQUIP). Alcohol Use Disorders Identification Test. Arch Intern Med 1998;158(16):1789-95.

15. Rollnick S, Heather N, Gold R, et al. Development of a short 'readiness to change' questionnaire for use in brief opportunistic interventions among excessive drinkers. $\mathrm{Br} \mathrm{J}$ Addict 1992; 87:743-54.

16. Holland RA, Evenson RC. Prediction of Readmission in a Cohort of Alcoholics: A 2-Year Follow-Up. Subst Use Misuse 1984; 19(5):585-90.

17. Center for Behavioral Health Statistics and Quality. (2011). Results from the 2010 National Survey on Drug Use and Health: Summary of national findings (HHS Publication No. SMA 11-4658, NSDUH Series H-41). Rockville, MD: Substance Abuse and Mental Health Services Administration.

\section{Declaration of interests}

None of the authors has a conflict of interest in relation to the project.

\section{Acknowledgements}

Dr Dhivya Raju performed the audit of ICD codes with alcohol related admissions. The data were collected by Dr Muhammad Salman, Dr Rahul Mudannayake, and Dr Gagan Swami.

The two item questionnaire was designed by Dr Rahul Mudannayake. The project was reviewed and supported by $\mathrm{Dr}$ Jonathan Sutton and Dr Waqar Ahmed. Members of the drug and alcohol team (Dr John Simms and Dr Jim O'Toole) provided information for the project and participated in the workshop. Dr Chrstian P Subbe acted as a mentor and performed the statistical analysis.

Cathrine Sutton reviewed the treatment recommendations for alcohol detoxification. Llinos Jones, on the acute medical unit helped with the dissemination of questionnaires and admission proformas.

\section{Ethical approval}

The project represents a service improvement project. Ethical approval was not required or requested. 